not greater than $\boldsymbol{\aleph}_{\alpha}$, it follows that a class of $\boldsymbol{\aleph}_{\alpha+1}$ of them has power $\boldsymbol{\aleph}_{\alpha+1}$. Such a class has at least one point in common with every perfect subset of $M$. Thus, there results the inequality

$$
\aleph_{\alpha+1} \leqq 2^{\aleph_{\alpha}} \text {. }
$$

The inequality in the opposite sense being well known, the generalized hypothesis of the continuum follows.

WAYNE UNIVERSITY

\title{
UNIQUENESS THEOREMS FOR RATIONAL FUNCTIONS ${ }^{1}$
}

\section{FRITZ HERZOG}

In his book on the theory of meromorphic functions, ${ }^{2} \mathrm{R}$. Nevanlinna proved a number of "uniqueness theorems." The most important of them ${ }^{3}$ states that if two functions $w=f(x)$ and $w=g(x)$, meromorphic in the whole $x$-plane, assume five values of $w$ (finite or infinite) at the same points $x$ they must be identical. If we understand by the distribution of a function $w=\phi(x)$ with respect to a given value of $w$ simply the set of all points $x$ where $\phi(x)$ assumes that value $w$, regardless of multiplicity, we may state the above theorem in the following way: Two meromorphic functions which have identical distributions with respect to five values of the dependent variable must be identical. In proving this theorem, Nevanlinna explicitly assumes the functions to be transcendental (i.e., not rational). ${ }^{3}$ It is trivial, however, that the theorem would apply to two rational functions $w=f(x)$ and $w=g(x)$ as well, which can be easily seen by considering the transcendental functions $w=f\left(e^{x}\right)$ and $w=g\left(e^{x}\right)$.

The example of the functions $w=e^{x}$ and $w=e^{-x}$, which have identical distributions with respect to the four values $w=1,-1,0, \infty$, shows that five is the smallest number for which the above-mentioned uniqueness theorem holds true. It will be shown in this paper that such is not the case for rational functions for which five may, indeed, be replaced by four. (See Theorem I.)

The question arises as to what can be said about two rational functions that have identical distributions with respect to only three

\footnotetext{
1 Presented to the Society, February 24, 1940.

${ }^{2}$ Rolf Nevanlinna, Le Théorème de Picard-Borel et la Thêrie des Fonctions Méromorphes, Paris, 1929.

${ }^{3}$ See loc. cit., p. 109.
} 
values of the dependent variable. ${ }^{4}$ It is the main object of this paper to study this problem.

It will be found that there exist pairs of rational functions which have identical distributions with respect to three values without being identical (Examples I, II, III). Furthermore, under certain restrictive conditions concerning, for instance, the degrees of the functions, uniqueness theorems for rational functions with identical distributions with respect to three values will be derived. (See Theorems II and III.)

Throughout the paper we shall use $x$ or $z$ for the independent complex variable and $w$ for the dependent complex variable. Unless otherwise stated, $x$ is to be restricted to finite values while $z$ and $w$ may assume also the value $\infty$.

As far as the variables $z$ and $w$ are concerned, we may subject them to linear substitutions without changing the character of the problem under consideration. This is due to the fact that a substitution $u=\left(a u_{1}+b\right) /\left(c u_{1}+d\right)$ with $a d-b c \neq 0$ gives a one-to-one correspondence between the $u$ and $u_{1}$ planes (both $u=\infty$ and $u_{1}=\infty$ being included). We shall sometimes make use of such linear substitutions without further explanation.

We finally remark that all functions mentioned in the paper are understood to be not constant, unless otherwise stated.

We consider two rational functions $w=f(x)$ and $w=g(x)$ which have identical distributions with respect to four values of $w$ and shall show that they must be identical. In accordance with what was said above, $x$ is restricted to finite values, that is, the two functions are assumed to have identical distributions with respect to the four values of $w$, only as far as finite values of $x$ are concerned, while the point $x=\infty$ is disregarded (except at certain points in the proof). We thus obtain a more general theorem.

THEOREM I. If $w=f(x)$ and $w=g(x)$ are two rational functions and if $w_{1}, w_{2}, w_{3}, w_{4}$ are four different values of $w$ that are assumed by the two functions at the same points $x$, then $f(x) \equiv g(x)$.

Proof. Let $m$ and $n$ be the degrees of $f(x)$ and $g(x)$, respectively; we may assume that $m \geqq n$. Let $w_{0}=f(\infty)$ where $w_{0}$ may be either dif-

${ }^{4}$ Cf. Nevanlinna's remark, loc. cit., p. 109: ". . . une fonction rationnelle est univoquement déterminée par trois distributions." In view of the results of this paper, this remark must be understood in such a way that identical distribution is to imply also equal multiplicities. Neither in Nevanlinna's uniqueness theorem nor in this paper is this the case. 
ferent from $w_{1}, w_{2}, w_{3}$, and $w_{4}$ or equal to one of them. In the latter case we may assume that $w_{0}=w_{1}$.

Now let $r_{k}$ be the actual number of finite $w_{k}$-points of $f(x)$ and $g(x)$ $(k=1,2,3,4)$, that is, their number not counting multiplicity. Let $m_{0}$ be the multiplicity of $w_{0}$ at $x=\infty$ and $m_{k}$ be the number of finite $w_{k}$-points of $f(x)(k=1,2,3,4)$, counting multiplicity. Then we have $m_{1}=m$ or $m_{0}+m_{1}=m$, according to whether $w_{0} \neq w_{1}$ or $w_{0}=w_{1}$. Hence, in any case,

$$
m_{0}+m_{1} \geqq m \text {. }
$$

Also, in any case,

$$
m_{2}=m_{3}=m_{4}=m .
$$

We may assume, without loss of generality, that $w_{0}, w_{1}, w_{2}, w_{3}$, and $w_{4}$ are all finite because a suitable linear substitution of $w$ will, if necessary, transform the $w_{k}$ into finite values.

Now let $f=F_{1} / F_{2}$ and $g=G_{1} / G_{2}$ be representations of $f$ and $g$ as reduced fractions of polynomials. We consider the functions

$$
\begin{aligned}
f^{\prime} & =\left(F_{2} F_{1}^{\prime}-F_{1} F_{2}^{\prime}\right) / F_{2}^{2}, \\
f-g & =\left(F_{1} G_{2}-F_{2} G_{1}\right) / F_{2} G_{2} .
\end{aligned}
$$

(3) is not identically zero, it is of degree at most $2 m$, it vanishes at $x=\infty$ with a multiplicity $m_{0}+1$ (which can be seen easily from the expansion of $f(x)$ in a power series about $x=\infty)$, and it has $m_{k}-r_{k}$ zeros, counting multiplicity, at the $r_{k}$ finite $w_{k}$-points of $f(x)(k=1,2$, $3,4)$. These facts yield

(5) $\left(m_{0}+1\right)+\left(m_{1}-r_{1}\right)+\left(m_{2}-r_{2}\right)+\left(m_{3}-r_{3}\right)+\left(m_{4}-r_{4}\right) \leqq 2 m$.

Using (1) and (2), we obtain from (5)

$$
4 m+1-\left(r_{1}+r_{2}+r_{3}+r_{4}\right) \leqq 2 m
$$

or

$$
r_{1}+r_{2}+r_{3}+r_{4} \geqq 2 m+1 .
$$

On the other hand, (4) is of degree at most $m+n \leqq 2 m$, it vanishes at the $r_{k}$ finite points $x$ where $f(x)=g(x)=w_{k}(k=1,2,3,4)$, and hence, by (6), it has at least $r_{1}+r_{2}+r_{3}+r_{4} \geqq 2 m+1$ zeros. Therefore, (4) must vanish identically, which proves the theorem.

That Theorem I does not hold when only three identical distributions are assumed can be seen from very simple examples, such as $w=f(x)=4 x /(x+1)^{2}$ and $w=g(x)=2 x /(x+1)$ which have identical distributions with respect to the three values $w=0, \infty, 1$, provided 
we consider only finite $x$. The value $w=0$, in particular, is assumed by $f(x)$ and $g(x)$ at $x=0$. If, however, $x$ were allowed to assume also the value $\infty$, the distributions of $f(x)$ and $g(x)$ with respect to $w=0$ would not be identical any more since $f(\infty)=0$ but $g(\infty) \neq 0$.

We shall, therefore, from now on allow the independent variable to assume also the value $\infty$ and study the problem of two rational functions $w=f(z)$ and $w=g(z)$ which have identical distributions with respect to three values of $w$.

We first consider the special case of polynomials. Since a polynomial assumes the value $w=\infty$ at $z=\infty$ only, the question is whether two polynomials with identical distributions with respect to two (finite) values of $w$ must be identical. The answer is given by the following theorem.

THEOREM II. If two polynomials $w=F(z)$ and $w=G(z)$ assume two finite values $w_{1}$ and $w_{2}\left(w_{1} \neq w_{2}\right)$ at the same points $z$, then $F(z) \equiv G(z)$.

Proof. Let $m$ and $n$ be the degrees of $F(z)$ and $G(z)$, respectively, let $m \geqq n$ and let $r_{k}$ be the actual number of $w_{k}$-points of $F(z)$ and $G(z)(k=1,2)$, not counting multiplicity. Then $F^{\prime}(z)$ is not identically zero, it is of degree $m-1$ and it has $m-r_{k}$ zeros, counting multiplicity, at the $r_{k}$ points where $F(z)=w_{k}(k=1,2)$. This gives $\left(m-r_{1}\right)$ $+\left(m-r_{2}\right) \leqq m-1$ or

$$
r_{1}+r_{2} \geqq m+1 \text {. }
$$

On the other hand, $F(z)-G(z)$ is of degree at most $m$ and it vanishes at the $r_{k}$ points where $F(z)=G(z)=w_{k}(k=1,2)$. Hence, by (7), it has at least $r_{1}+r_{2} \geqq m+1$ zeros and must, therefore, vanish identically. This proves the theorem.

By subjecting both $z$ and $w$ to linear substitutions the two polynomials become two rational functions which have the property that there is one value of $w$ which is assumed by both functions at one and the same point $z$ only. Thus, according to what was said in the introduction, Theorem II yields immediately the following corollary.

CoRollary. If two rational functions $w=f(z)$ and $w=g(z)$ assume three different values $w_{1}, w_{2}, w_{3}$ at the same points $z$ and if one of these three values is assumed at one point $z$ only, then $f(z) \equiv g(z)$.

The corollary just proved is one of several similar statements that will be derived in the following. These statements give sufficient conditions under which two rational functions with identical distributions with respect to three values must be identical. It will simplify the language if we put these statements in the form of necessary con- 
ditions which two different rational functions have to satisfy if they have identical distributions with respect to three values of the dependent variable.

Thus we consider two rational functions $w=f(z)$ and $w=g(z)$ which assume three different values $w_{1}, w_{2}, w_{3}$ at the same points $z$, which we shall call $z_{k s}\left(k=1,2,3 ; s=1,2, \cdots, r_{k}\right.$; all $z_{k s}$ different $)$. Furthermore, we assume that the degrees of $f(z)$ and $g(z)$ are $m$ and $n$, respectively, and that $m \geqq n$. Finally, let $m_{k s}$ and $n_{k s}$ be the multiplicities with which $w=f(z)$ and $w=g(z)$, respectively, assume the value $w=w_{k}$ at $z=z_{k s}\left(m_{k s} \geqq 1, n_{k s} \geqq 1\right)$. It will be convenient to enter these data in the following table.

\begin{tabular}{|c|c|c|c|}
\hline \multirow{2}{*}{$\begin{array}{l}\text { Value } \\
\text { of w }\end{array}$} & \multirow{2}{*}{$\begin{array}{l}\text { Distribution of } f(z) \\
\text { and } g(z) \text { with } \\
\text { respect to } w_{k}\end{array}$} & \multicolumn{2}{|c|}{ Multiplicities at $z_{k s}$ of } \\
\hline & & $f(z)$ & $g(z)$ \\
\hline$w_{1}$ & $z_{11}, z_{12}, \cdots, z_{1 r_{1}}$ & $m_{11}, m_{12}, \cdots, m_{1 r_{1}}$ & $n_{11}, n_{12}, \cdots, n_{1 r_{1}}$ \\
\hline$w_{2}$ & $z_{21}, z_{22}, \cdots, z_{2 r_{2}}$ & $m_{21}, m_{22}, \cdots, m_{2 r_{2}}$ & $n_{21}, n_{22}, \cdots, n_{2 r_{2}}$ \\
\hline$w_{3}$ & $z_{31}, z_{32}, \cdots, z_{3 r_{8}}$ & $m_{31}, m_{32}, \cdots, m_{3 r_{8}}$ & $n_{31}, n_{32}, \cdots, n_{3 r_{3}}$ \\
\hline
\end{tabular}

We note that for $k=1,2,3$

$$
\begin{aligned}
m_{k 1}+m_{k 2}+\cdots+m_{k r_{k}} & =m, \\
n_{k 1}+n_{k 2}+\cdots+n_{k r_{k}} & =n .
\end{aligned}
$$

THEOREM III. If $w=f(z)$ and $w=g(z)$ are two rational functions with identical distributions with respect to three values of $w$ and if $w_{k}, r_{k}, z_{k s}$, $m, n, m_{k s}, n_{k s}$ are as defined above, then the following are necessary conditions for $f(z) \not \equiv g(z)$ :

$$
\sum_{k=1}^{3} \sum_{s=1}^{r_{k}} \min \left(m_{k s}, n_{k s}\right) \leqq m+n,
$$

$$
r_{1}+r_{2}+r_{3} \geqq m+2,
$$

$$
2 \leqq r_{k} \leqq n,
$$$$
\text { for } k=1,2,3 \text {, }
$$

$$
m+n \geqq 6 \text {, }
$$$$
m \leqq 3 n-2 \text {, }
$$

(f) $\quad\left(m_{k 1}, m_{k 2}, \cdots, m_{k r_{k}}\right)=\left(n_{k 1}, n_{k 2}, \cdots, n_{k r_{k}}\right)$ for at most one $k^{.5}$

Proof. For the proof of conditions (a) to (e) we assume that the three $w_{k}$ as well as all the $z_{k s}$ are finite numbers.

${ }^{5}$ This equation is to be considered an identity of two vectors, that is, $m_{k 1}=n_{k 1}$, $m_{k 2}=n_{k 2}, \cdots, m_{k r}=n_{k r}$. 
(a) The function $f(z)-g(z)$ (see (4) above) is not identically zero, it is of degree at most $m+n$, it has a zero at $z=z_{k s}$ the multiplicity of which is at least $\min \left(m_{k s}, n_{k s}\right)$, and it has, therefore, at least $\sum_{k=1}^{3} \sum_{s=1}^{r_{k}} \min \left(m_{k s}, n_{k s}\right)$ zeros, counting multiplicity. Thus (a) must hold.

(b) The function $f^{\prime}(z)$ (see (3) above) is not identically zero and the numerator $F_{2} F_{1}^{\prime}-F_{1} F_{2}^{\prime}$ is of degree at most $2 m-2$. At the points $z_{k s}\left(s=1,2, \cdots, r_{k}\right) f^{\prime}(z)$ has $m-r_{k}$ zeros, counting multiplicities $(k=1,2,3)$, hence $f^{\prime}(z)$ has at least $\left(m-r_{1}\right)+\left(m-r_{2}\right)+\left(m-r_{3}\right)$ finite zeros which must be zeros of the numerator $F_{2} F_{1}^{\prime}-F_{1} F_{2}^{\prime}$. This gives

$$
3 m-\left(r_{1}+r_{2}+r_{3}\right) \leqq 2 m-2,
$$

from which (b) follows.

(c) The inequality $r_{k} \geqq 2$ (for $k=1,2,3$ ) is easily seen to be identical with the corollary, proved above. That $r_{k} \leqq n$ (for $k=1,2,3$ ) follows at once from (9) and $n_{k s} \geqq 1$.

(d) From inequality (a) and the fact that $\min \left(m_{k s}, n_{k s}\right) \geqq 1$ (for $k=1,2,3$ and $\left.s=1,2, \cdots, r_{k}\right)$ we conclude

$$
r_{1}+r_{2}+r_{3} \leqq m+n \text {. }
$$

(10) together with $r_{k} \geqq 2$ from (c) yields (d).

(e) From (b) we conclude

$$
m \leqq r_{1}+r_{2}+r_{3}-2 .
$$

(11) together with $r_{k} \leqq n$ from (c) yields (e).

(f) Suppose we should have $\left(m_{k 1}, m_{k 2}, \cdots, m_{k r_{k}}\right)=\left(n_{k 1}, n_{k 2}, \cdots, n_{k r_{k}}\right)$ for two values of $k$, say $k=1$ and $k=2$. We may then assume that $w_{1}=0$ and $w_{2}=\infty$. Thus $f(z)$ and $g(z)$ have the same zeros and the same poles, including their multiplicities, and hence $f(z) \equiv c g(z)$ with $c=$ const. Since $f\left(z_{31}\right)=g\left(z_{31}\right)=w_{3}\left(w_{3} \neq 0, \neq \infty\right)$ we have $c=1$ which contradicts $f(z) \not \equiv g(z)$. This proves condition $(\mathbf{f})$.

We remark that the inequalities (a) to (e) in the form in which they are written above cannot be improved. Indeed, in Example I below both sides of these inequalities are actually equal. As to condition (f), the relation $\left(m_{k 1}, m_{k 2}, \cdots, m_{k r_{k}}\right)=\left(n_{k 1}, n_{k 2}, \cdots, n_{k r_{k}}\right)$ may actually be satisfied for one $k$, as shown by Example III.

We shall now give three examples of pairs of rational functions with identical distributions with respect to three values of the dependent variable. We shall briefly describe the procedure for finding such examples. For notations in what follows, we refer to the above table.

We select degrees $m$ and $n$ with $m \geqq n$ and satisfying the conditions of Theorem III. Then we select positive integers $r_{k}, m_{k s}$ and $n_{k s}$, com- 
patible with (8) and (9) as well as with all the conditions of Theorem III. By the principle of linear substitutions of $w$ and $z$ we may then assign any three arbitrary values to the three $w_{k}$, say $w_{1}=0, w_{2}=\infty$, $w_{3}=1$, and three arbitrary values to three arbitrarily selected ones among the $z_{k s}$, say $z_{11}=1, z_{21}=\infty, z_{22}=0$. (Observe that, by condition (c), $r_{2} \geqq 2$.) With the other $z_{k s}$ as unknowns and with two unknown constants $a$ and $b$ we have then in view of the distributions with respect to $w_{1}=0$ and $w_{2}=\infty$

$$
\begin{aligned}
& f(z)=\left[(z-1)^{m_{11}} \prod_{s=2}^{r_{1}}\left(z-z_{1 s}\right)^{m_{1 s}}\right] /\left[a z^{m_{22}} \prod_{s=3}^{r_{2}}\left(z-z_{2 s}\right)^{m_{2 s}}\right], \\
& g(z)=\left[(z-1)^{n_{11}} \prod_{s=2}^{r_{1}}\left(z-z_{1 s}\right)^{n_{1 s}}\right] /\left[b z^{n_{22}} \prod_{s=3}^{r_{2}}\left(z-z_{2 s}\right)^{n_{2 s}}\right]
\end{aligned}
$$

where the product in the denominator to the right of (12) and (13) is to be unity if $r_{2}=2$. Now the distribution with respect to $w_{3}=1$ gives

$$
\begin{aligned}
& (z-1)^{m_{11}} \prod_{s=2}^{r_{1}}\left(z-z_{1 s}\right)^{m_{1}}-a z^{m_{22}} \prod_{s=3}^{r_{2}}\left(z-z_{2 s}\right)^{m_{2 s}}=\prod_{s=1}^{r_{3}}\left(z-z_{3 s}\right)^{m_{3 s}} \\
& (z-1)^{n_{11}} \prod_{s=2}^{r_{1}}\left(z-z_{1 s}\right)^{n_{1 s}}-b z^{n_{22}} \prod_{s=3}^{r_{2}}\left(z-z_{2 s}\right)^{n_{2 s}}=\prod_{s=1}^{r_{3}}\left(z-z_{3 s}\right)^{n_{3 s}}
\end{aligned}
$$

If now the coefficients of equal powers of $z$ on both sides of (14) and (15) are put equal to one another we obtain $m+n$ equations for the $r_{1}+r_{2}+r_{3}-1$ unknowns $z_{k s}, a$ and $b .{ }^{6}$ Each solution of these equations in which the $z_{k s}$ are different from each other and from 0 and 1 and in which $a$ and $b$ are different from 0 corresponds to a pair of functions of the desired type.

By condition (c) of Theorem III, the smallest value for $n$ is 2 . If $n=2$ we have, by conditions (d) and (e), $m=4$. The above procedure then leads to the following example:

EXAMPLE I. $f(z)=(z-1)^{3}(z+3) / 16 z, g(z)=(z-1)(z+3) / 4 z$.

\begin{tabular}{|c|c|c|c|}
\hline \hline$w_{k}$ & $z_{k s}$ & $m_{k s}$ & $n_{k s}$ \\
\hline 0 & $1,-3$ & 3,1 & 1,1 \\
$\infty$ & $\infty, 0$ & 3,1 & 1,1 \\
1 & $-1,3$ & 3,1 & 1,1 \\
\hline
\end{tabular}

${ }^{6}$ From (10) it follows that we have at least one more equation than unknowns. 
We remark without proof that the above pair is essentially the only pair with $m=4$ and $n=2$. That is to say that first no other combinations of $m_{k s}$ and $n_{k s}$ are possible (except permutations of the above) and that secondly all other pairs of degrees $m=4$ and $n=2$ are obtained from the above by a suitable linear substitution of $w$ and $z$.

The next lowest degrees, according to condition (d), are $m=3, n=3$. These values lead to the following example.

EXAMPLE II.

$$
\begin{array}{r}
\left.f(z)=(z-1)^{2}(z+2) / 3\left(\rho^{2}-1\right) z, \quad g(z)=z-1\right)(z+2)^{2} / 3(1-\rho) z^{2}, \\
\rho=-\frac{1}{2}+\frac{1}{2} i 3^{1 / 2} .
\end{array}
$$

\begin{tabular}{|c|c|c|c|}
\hline \hline$w_{k}$ & $z_{k s}$ & $m_{k s}$ & $n_{k s}$ \\
\hline 0 & $1,-2$ & 2,1 & 1,2 \\
$\infty$ & $\infty, 0$ & 2,1 & 1,2 \\
1 & $\rho,-2 \rho$ & 2,1 & 1,2 \\
\hline
\end{tabular}

Example II is again essentially the only pair of functions with $m=3, n=3$ in the same sense as Example I above.

The case $m=4, n=4$ leads to two interesting examples. One of them is the following.

EXAMPLE III. $f(z)=(z-1)^{3}(z+1) / 4 z, g(z)=(z-1)(z+1)^{3} / 4 z^{3}$.

\begin{tabular}{|c|c|c|c|}
\hline \hline$w_{k}$ & $z_{k s}$ & $m_{k s}$ & $n_{k s}$ \\
\cline { 2 - 4 } & $1,-1$ & 3,1 & 1,3 \\
$\infty$ & $\infty, 0$ & 3,1 & 1,3 \\
1 & $i,-i, 1+2^{1 / 2}, 1-2^{1 / 2}$ & $1,1,1,1$ & $1,1,1,1$ \\
\hline
\end{tabular}

In this example the relation of condition ( $f$ ) of Theorem III is actually satisfied for one $k$, namely, $k=3$.

Another example with $m=4, n=4$ can be obtained from Example I by replacing $z$ by $-3 / z$ in the function $f(z)$. It is easily seen that $f(-3 / z)$ and $f(z)$ form a pair of functions of the desired type. Thus the three functions ${ }^{7} f(z), f(-3 / z)$ and $g(z)$ of Example I form a triplet of rational functions with identical distributions with respect to three values of the dependent variable.

${ }^{7}$ Note that $g(-3 / z) \equiv g(z)$. 
The last mentioned example raises the question as to whether there could be an infinite number of rational functions which have given distributions with respect to three given values. This question is answered as follows.

THEOREM IV. There can be only a finite number of rational functions which have given distributions with respect to three given values of the dependent variable.

Proof. Let $z_{k s}$ be the given distributions with respect to the given values $w_{k}$ (see table preceding Theorem III). Let $w=f(z)$ be any rational function having these distributions, let $m$ be its degree and $m_{k s}$ be its multiplicity at $z=z_{k s}$. Then, by the proof of condition (b) in Theorem III, $m$ cannot exceed $r_{1}+r_{2}+r_{3}-2$. This leaves only a finite number of values for $m$, hence by (8) there is only a finite number of possible combinations of $m_{k s}$. Each such combination can, according to condition (f) of Theorem III, lead to at most one function $f(z)$. This proves the theorem.

It may be noted in conclusion that any pair of functions $w=f(z)$ and $w=g(z)$ of the above-mentioned type leads to infinitely many more pairs of the same kind, other than those obtained by linear substitutions of $z$ and $w$. For, if $\phi(z)$ is any rational function whatever, then $w=f(\phi(z))$ and $w=g(\phi(z))$ have again identical distributions with respect to the same three values of $w$ as the original functions $w=f(z)$ and $w=g(z)$. This shows, in particular, that the degrees $m$ and $n$ of such pairs of functions may exceed any given number.

Cornell University 